\title{
34. COMMISSION DE LA PARALLAXE SOLAIRE
}

\section{President: M. Dyson, Astronomer Royal of England, Royal Observatiry, Greenwich, England.}

MEmBREs: MM. Esclangon, Hinks, Jones and Schlesinger.

I. A report of the progress of the Meridian observations has been given in Section 8.

2. The question is also raised as to whether it is desirable to supplement these observations by a number of photographs of large fields, particularly for the region traversed by Eros in the interval from Jan. I to Feb. 28. The positions of all stars down to rom.o photographic, on a band within $I^{\circ}$ of the path of Eros, will probably furnish all the necessary comparison stars.

3. Eros will be of photographic magnitude, $7^{\mathrm{m} \cdot 5^{-8}} 8^{\mathrm{m} \cdot 0}$ from Jan. I to Feb. 28 , when its parallax is greatest. For photographic observations, it will probably be necessary to use a rotating shutter and reduce the magnitude to about $9^{\mathrm{m} \cdot 5}$ photographic.

4. It would seem that exposures of about $\mathrm{I}$ minute will be suitable as a working programme for Astrographic telescopes with a field of $2^{\circ}$. The planet will have trailed about $2 \frac{1}{2}$ " in one minute: it will be most convenient to have it in the middle of the field.

5. Attention will need to be given to the colours of the reference stars. The brighter stars are to be found in the Draper Catalogue, so their colours can be inferred. Any determinations of spectral type or colour of reference stars will be of value. It will be well to reject comparison stars of extreme colour.

6. Determinations will be required of the colour of Eros. The uncertainty owing to the possible difference of colour between the planet and comparison stars is the weakest point in the rgoo-Igor determinations of the Solar parallax.

7. An accurate Ephemeris of the planet will be required as soon as observations permit of its construction.

This opposition offers opportunities for the comparison of observations at Northern and Southern Observatories, as well as the employment of the diurnal method.

The points referred to above may serve to indicate lines on which discussion should go in order to secure the co-operation of different classes of work.

\author{
F. W. Dyson \\ President of the Commission
}

